\title{
TABELA DE VIDA DE FERTILIDADE DE Trichogramma pratissolii Querino \& Zucchi, 2003 (Hymenoptera: Trichogrammatidae) EM HOSPEDEIROS ALTERNATIVOS, SOB DIFERENTES TEMPERATURAS
}

\author{
Fertility life table of Trichogramma pratissolii Querino \& Zucchi, 2003 (Hymenoptera: \\ Trichogrammatidae) in alternative hosts, under different temperatures
}

\author{
Hugo Bolsoni Zago ${ }^{1}$, Dirceu Pratissoli², Reginaldo Barros ${ }^{3}$, Manoel Guedes Côrrea Gondim Junior ${ }^{3}$
}

\begin{abstract}
RESUMO
A espécie, Trichogramma pratissolii Querino \& Zucchi (Hymenoptera: Trichogrammatidae) foi recentemente coletada em plantios de abacate, Persea americana Mill. (Lauraceae), e pode representar uma alternativa no controle biológico de lepidópteros pragas dessa cultura. Assim, objetivou-se neste trabalho avaliar o desempenho de T. pratissolii criado em ovos de Anagasta kuehniella (Zeller) (Lepidoptera: Pyralidae) e Corcyra cephalonica (Stainton) (Lepidoptera: Pyralidae), sob diferentes temperaturas utilizando tabelas de vida de fertilidade. A criação de A. kuehniella foi mantida com dieta à base de farinha de trigo integral, milho e levedura de cerveja, enquanto para C. cephalonica foi utilizada dieta à base de farelo de arroz, levedura de cerveja e açúcar. T. pratissolii foi criado nos hospedeiros, utilizando-se a técnica de colagem de ovos em cartolina azul. Foram determinadas a duração média da geração (T), taxa líquida de reprodução $\left(\mathrm{R}_{\mathrm{o}}\right)$, razão infinitesimal de aumento $\left(\mathrm{r}_{\mathrm{m}}\right)$ e razão infinita de aumento $(\lambda)$ para as temperaturas de $15 ; 21 ; 24$; $27 ; 30$ e $33 \pm 1^{\circ} \mathrm{C}, \operatorname{com} 70 \pm 10 \%$ de umidade relativa e fotofase de 14 horas. A melhor condição térmica para desenvolvimento de $T$. pratissolii em A. kuehniella e C. cephalonica foi a $27^{\circ} \mathrm{C}$, enquanto o melhor hospedeiro nessa temperatura é C. cephalonica.
\end{abstract}

Termos para indexação: Controle biológico, parasitóide de ovos, Trichogrammatidae.

\begin{abstract}
The specie, Trichogramma pratissolii Querino \& Zucchi (Hymenoptera: Trichogrammatidae) was recently collected in avocado plantations, Persea americana Mill. (Lauraceae), and therefore might be an alternative biological control agent for lepidopteran pests in this crop. Thus, this work evaluated the performance of T. pratissolii parasitizing eggs of Anagasta kuehniella Zeller (Lepidoptera: Pyralidae) and Corcyra cephalonica Stainton (Lepidoptera: Pyralidae), under different temperature regimes through fertility life table method. The host A. kuehniella was reared using diet constituted of whole wheat meal, corn meal and yeast, while C. cephalonica was reared with rice meal, yeast and sugar. Eggs of both hosts were offered to T. pratissolii pasted in blue cards. Mean generation time $(T)$, net reproductive rate $\left(\mathrm{R}_{\mathrm{o}}\right)$, intrinsic rate of population increase $\left(\mathrm{r}_{\mathrm{m}}\right)$ and finite rate of increase $(\lambda)$ were estimated for parasitoid reared under $15 ; 21 ; 24 ; 27 ; 30$ and $33 \pm 1^{\circ} \mathrm{C}, 70 \pm 10 \%$ relative humidity and 14 hours of photoperiod. The best thermal conditions to T. pratissolii develop parasitizing A. kuehniella and C. cephalonica was $27^{\circ} \mathrm{C}$, and under this temperature eggs of the later host produced the better results.
\end{abstract}

Index terms: Biological control, egg parasitoids, Trichogrammatidae.

(Recebido em 17 de outubro de 2006 e aprovado em 22 de fevereiro de 2007)

\section{INTRODUÇÃO}

O gênero Trichogramma compreende os parasitóides de ovos mais estudados mundialmente e muito utilizados em programas de controle biológico de pragas agrícolas, principalmente as pertencentes à ordem Lepidoptera (HASSAN, 1997). São bastante eficientes podendo ser produzidos em hospedeiros alternativos, característica que possibilita a viabilidade de sua utilização (PARRA, 1997).
Dominar a produção em laboratório representa a etapa básica para um programa de controle biológico com a utilização desses entomófagos, pois os parâmetros biológicos desses insetos são muitos afetados pelo hospedeiro e temperatura utilizados nas criações massais, podendo dessa maneira comprometer o sucesso do controle biológico, já que inimigos naturais produzidos de forma inadequada, resultarão em indivíduos de menor potencial de eficiência (PREZOTTI \& PARRA, 2002).

\footnotetext{
'Doutor em Entomologia Agrícola - Departamento de Agronomia/DEPA - Universidade Federal Rural de Pernambuco/UFRPE - Rua Dom Manoel de Medeiros, s/n - Dois Irmãos - 52171-900 - Recife, PE - zagoh@hotmail.com

2Doutor em Entomologia - Centro de Ciências Agrárias/CCA - Universidade Federal do Espírito Santo/UFES - Alto Universitário, s/n - Cx. P. 16 - 29500-000 _ Alegre, ES - pratissoli@cca.ufes.br

${ }^{3}$ Doutores em Entomologia - Departamento de Agronomia/DEPA - Universidade Federal Rural de Pernambuco/UFRPE - Rua Dom Manoel de Medeiros, s/n - Dois Irmãos - 52171-900 - Recife, PE - rbarros@depa.ufrpe.br; guedes@depa.ufrpe.br
} 
Sempre que uma nova espécie e/ou linhagem é encontrada, como T. pratissolii (QUERINO \& ZUCCHI, 2003), estudos básicos de laboratório são obrigatórios para auxiliar trabalhos futuros para uma utilização racional dessa espécie em programas de controle biológico (PRATISSOLI \& PARRA, 2000).

A utilização de modelos matemáticos, como o estudo das tabelas de vida de fertilidade fornecem subsídios para comparar parâmetros biológicos de insetos sujeitos à variações de caráter biótico e abiótico do ambiente (SILVEIRA NETO et al., 1976). As tabelas são muito utilizadas para o entendimento de dinâmicas populacionais, avaliando a performance biológica e comparando o desenvolvimento de espécies e/ou linhagens de insetos (PRATISSOLI \& PARRA, 2000).

Desse modo, objetivou-se neste trabalho, avaliar o desempenho de $T$. pratissolii, criado em ovos de $A$. kuehniella e $C$. cephalonica, sob diferentes temperaturas, com base nos estudos das tabelas de vida de fertilidade.

\section{MATERIAL E MÉTODOS}

O experimento foi desenvolvido no Laboratório de Entomologia do Centro de Ciências Agrárias da Universidade Federal do Espírito Santo (CCA-UFES), sendo constituído das seguintes etapas:

Criação dos Hospedeiros A. kuehniella e $C$. cephalonica. A metodologia empregada na criação de $A$. kuehniella e C. cephalonica foi a de PARRA (1997), utilizando uma dieta à base de farinha de trigo integral $(60 \%)$ e de milho (37\%) e levedura de cerveja $(3 \%)$ para $A$. kuehniella e uma dieta à base de farelo de arroz (94\%), levedura de cerveja (3\%) e açúcar (3\%) para C. cephalonica (BERNARDI et al., 2000).

Criação de $\boldsymbol{T}$. pratissolii. Esta espécie, que estava sendo anteriormente criada em ovos de A. kuehniella, foi criado por três gerações sucessivas em ovos da traça-dascrucíferas Plutella xylostella (Linneus, 1758) (Lepidoptera: Plutellidae), na tentativa de se eliminar qualquer efeito do condicionamento ao hospedeiro de criação. A partir dos descendentes do T. pratissolii, provenientes dos ovos de P. xylostella, iniciaram-se duas criações distintas desse parasitóide, uma mantida no hospedeiro A. kuehniella e outra mantida em C. cephalonica.

Para a manutenção de $T$. pratissolii nos hospedeiros alternativos foi adotada a técnica desenvolvida por PARRA (1997) oferecendo-se ovos dos hospedeiros A. kuehniella e $C$. cephalonica, colados em retângulos de cartolina azul celeste, com goma arábica. Os ovos dos hospedeiros foram previamente inviabilizados em lâmpada germicida. As cartelas com os ovos dos hospedeiros alternativos foram inseridas em recipientes de vidro, dentro dos quais encontravam-se adultos de T. pratissolii, por $24 \mathrm{~h}$. As cartelas com os ovos parasitados foram armazenadas em sala climatizada com temperatura de $25 \pm 1^{\circ} \mathrm{C}$, umidade relativa de $70 \pm 10 \%$ e fotofase de $14 \mathrm{~h}$.

Tabelas de vida de fertilidade de $T$. pratissolii em A. kuehniella e C. cephalonica. Para cada hospedeiro, 15 cartelas de cartolina azul celeste de 2,5 x 0,3 cm contendo 40 ovos, foram individualizadas em tubos de Duran, fechados com filme plástico PVC. Em cada tubo, foram introduzidas 4 fêmeas (idade 0-12 horas) de T. pratissolii, proveniente de cada hospedeiro, permitindo o parasitismo em câmaras climatizadas, reguladas a $15 ; 21 ; 24 ; 27 ; 30$ e 33 $\pm 1^{\circ} \mathrm{C}, 70 \pm 10 \%$ de umidade relativa (UR) e fotofase de 14 horas. Após 5 horas de parasitismo, as fêmeas foram retiradas com auxílio de pincel e os tubos foram mantidos nas mesmas condições. As observações foram realizadas diariamente para determinação do período de desenvolvimento, porcentagem de emergência e razão sexual em cada temperatura e hospedeiros estudados.

A capacidade de parasitismo também foi determinada nas temperaturas e hospedeiros mencionados anteriormente. Para cada temperatura e hospedeiro, 15 fêmeas (idade 0-6 horas) foram isoladas em tubos de Duran fechados com filme plástico PVC. Cartelas de cartolina azul celeste de 2,5 x 0,3 cm com 40 ovos (idade 0-24 horas) foram oferecidas a cada 24 horas, até a constatação da morte da fêmea de T. pratissolii. As cartelas com ovos parasitados, provenientes de cada tratamento, foram acondicionadas em sacos plásticos de $23,0 \times 4,0 \mathrm{~cm} \mathrm{e}$ mantidas nas mesmas condições.

Os seguintes parâmetros foram avaliados: número médio de ovos parasitados diariamente, total de ovos parasitados, longevidade das fêmeas, razão sexual e viabilidade do parasitismo.

As tabelas de vida de fertilidade de $T$. pratissolii foram calculadas através do software SAS baseado na estimativa de Jackknife, sendo os seguintes parâmetros determinados: duração média da geração $(\mathrm{T})$, taxa líquida de reprodução (Ro), razão infinitesimal de aumento (rm) e razão infinita de aumento $(\lambda)$.

\section{RESULTADOS E DISCUSSÃO}

As durações médias entre gerações (T) foram inversamente proporcionais à variação da temperatura de 15 a $33^{\circ} \mathrm{C}$, exceto para o desenvolvimento do parasitóide em ovos de C. cephalonica, entre 30 e $33^{\circ} \mathrm{C}$ (6,7 - 6,7 dias), quando os valores se estabilizaram (Tabela 1). A duração média das gerações de $T$. pratissolii entre os hospedeiros testados diferiu nas temperaturas $21 ; 27 ; 30$ e $33^{\circ} \mathrm{C}$, sendo maior quando o parasitóide se desenvolveu em ovos de $C$. cephalonica, exceto a $30^{\circ} \mathrm{C}$. 
A taxa líquida de reprodução (Ro) apresentou os maiores valores na faixa de temperatura de 21 a $27^{\circ} \mathrm{C}$ para A. kuehniella, enquanto para C. cephalonica os maiores valores se encontram na faixa de temperatura entre $21 \mathrm{e}$ $30^{\circ} \mathrm{C}$. Houve diferença na taxa líquida de reprodução, entre os hospedeiros testados, apenas na temperatura de 27 e $33^{\circ} \mathrm{C}$, sendo que a $27^{\circ} \mathrm{C}$ foi maior o número de fêmeas adicionadas a cada geração no hospedeiro C. cephalonica, e a $33^{\circ} \mathrm{C}$ foi maior, quando o parasitóide se desenvolveu em ovos de A. kuehniella (Tabela 1).

A razão infinitesimal de aumento ( $\mathrm{rm})$ apresentou crescimento com o aumento da temperatura até $27^{\circ} \mathrm{C}$ e a partir dessa, os valores estabilizaram-se, quando o hospedeiro foi A. kuehniella. Em C. cephalonica, a razão infinitesimal de aumento (rm), também cresceu com o aumento da temperatura até $27^{\circ} \mathrm{C}$, não diferindo de $30^{\circ} \mathrm{C}$, entretanto a $33^{\circ} \mathrm{C}$ ocorreu decréscimo. Entre os hospedeiros estudados, diferenças significativas ocorreram na razão infinitesimal de aumento apenas a 21 e $33^{\circ} \mathrm{C}$, sendo os maiores valores em ovos de A. kuehniella (Tabela 1).

A razão finita de aumento $(\lambda)$ apresentou crescimento diretamente proporcional com a amplitude térmica estudada, variando de 1,07 a 1,52 descendentes/ fêmea/dia em ovos de $A$. kuehniella entre $15^{\circ} \mathrm{C}$ e $27^{\circ} \mathrm{C}$, não havendo diferença entre essa última temperatura e as de $30^{\circ} \mathrm{C}$ e $33^{\circ} \mathrm{C}$. Para C. cephalonica, a razão finita de aumento cresceu de 1,06 a 1,55 descendentes/fêmea/dia entre as temperaturas de $15^{\circ} \mathrm{C}$ e $27^{\circ} \mathrm{C}$, não havendo diferença entre essa última e $30^{\circ} \mathrm{C}$, contudo, na temperatura de $33^{\circ} \mathrm{C}$ houve uma redução significativa desse valor (Tabela 1).

Diversos estudos corroboram os resultados encontrados nessa pesquisa, onde a temperatura representou o principal fator que interfere na duração do ciclo de Trichogramma spp. (CALVIN et al., 1984; BLEICHER \& PARRA, 1990; PEREIRA et al., 2004). Vários trabalhos, que avaliaram tabelas de vida de fertilidade, demonstraram que o tempo médio entre gerações é inversamente proporcional ao aumento da temperatura ambiente (PRATISSOLI \& PARRA, 2000; PRATISSOLI et al., 2004b).

Além da temperatura, variações nutricionais dos hospedeiros alternativos utilizados na criação de $T$. pratissolii também contribuíram para alterações nos parâmetros de tabela de vida de fertilidade de T. pratissolii, fato esse já relatado em outras pesquisas, com utilização de outras espécies de Trichogramma (PRATISSOLI \& PARRA, 2000; ZHANG et at., 2001; PRATISSOLI et al., 2004a; PRATISSOLI et al., 2004b).

O uso de tabela de vida de fertilidade representa uma ferramenta importante para o controle biológico, pois através dela é possível maximizar a criação do parasitóide

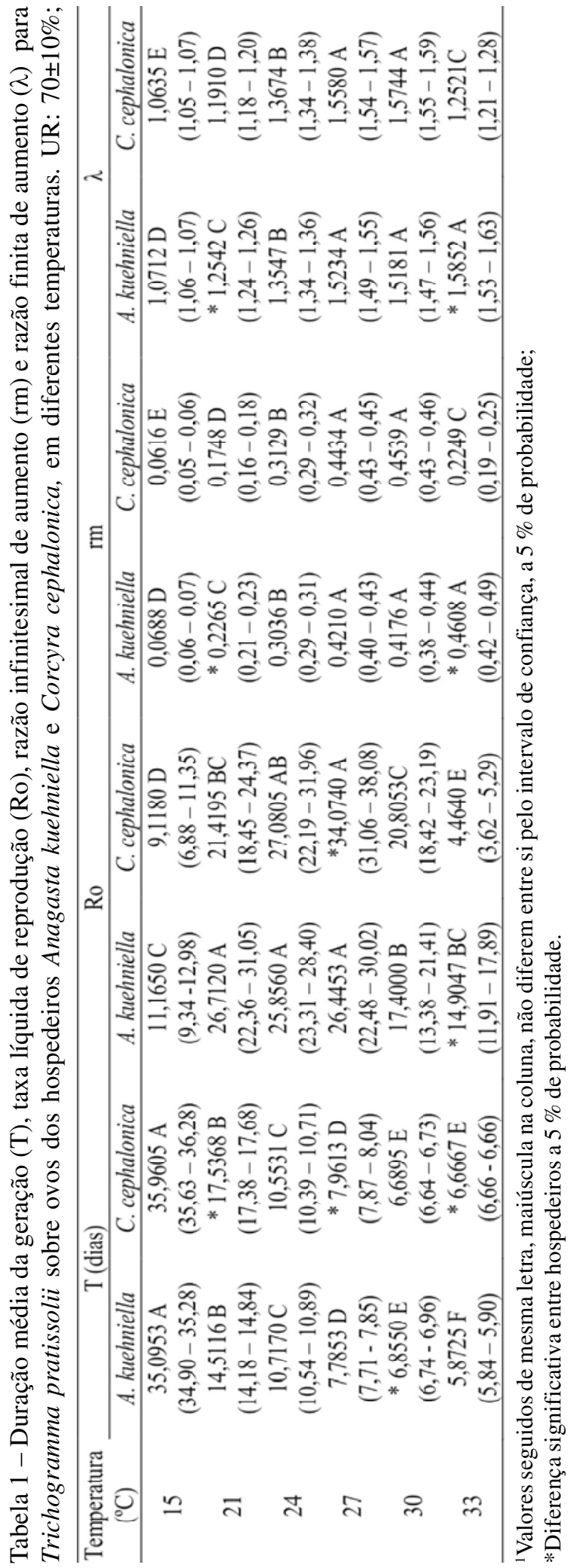


em laboratório, mantendo a criação de Trichogramma spp. em condições ideais de nutrição e temperatura para desenvolvimento e reprodução, produzindo assim fêmeas de alto desempenho, para uso no controle biológico.

\section{CONCLUSÕES}

A melhor condição térmica para desenvolvimento de T. pratissolii em A. kuehniella e C. cephalonica foi a $27^{\circ} \mathrm{C}$. Os resultados encontrados demonstram que $C$. cephalonica é melhor hospedeiro para a criação do parasitóide nessa temperatura, quando comparado a A. kuehniella.

\section{AGRADECIMENTOS}

A Dr ${ }^{a}$ Ranyse Barbosa Querino da Silva da EMBRAPA, Roraima-RR pela identificação da espécie Trichogramma pratissolii. À Coordenação de Aperfeiçoamento de Pessoal de Nível Superior (CAPES), por ter concedido a bolsa de estudos ao primeiro autor, à Universidade Federal Rural de Pernambuco (UFRPE) e ao Laboratório de Entomologia do Centro de Ciências Agrárias da Universidade Federal do Espírito Santo (CCA-UFES), por permitirem o desenvolvimento dessa pesquisa.

\section{REFERÊNCIAS BIBLIOGRÁFICAS}

BERNARDI, E. B.; HADDAD, M. L.; PARRA, J. R. P. Comparasion of artificial diets or rearing Corcyra cephalonica (Stainton, 1865) (Lep.: Pyralidae) for Trichogramma Mass production. Revista Brasileira de Biologia, São Carlos, v. 60, n 1, p. 45-52, 2000.

BLEICHER, E. ; PARRA, J. R. P. Espécies de Trichogramma parasitóides de Alabama argillacea. Determinação das exigências térmicas de três populações. Pesquisa Agropecuária Brasileira. Brasília, v.25, n. 1, p.215-219, 1990.

CALVIN, D. D.; KNAPP M. C.; WELCH, S. M.; POSTON, F. L. ; ELZINGA, R. J. Impact of environmental factors on Trichogramma pretiosum reader on southwestern corn borer eggs. Environment Entomology, Lanham, v.13, n. 3, p.774-780, 1984.

HASSAN, S. A. Seleção de espécies de Trichogramma para uso em programas controle biológico. In: PARRA, J. R. P.; ZUCCHI, R. A. (eds). Trichogramma e o Controle Biológico Aplicado. Piracicaba: FEALQ, 1997. p. 183-206.

PARRA, J. R. P.Técnicas de criação de Anagasta kuehniella, hospedeiro alternativo para produção de Trichogramma. In: PARRA, J. R. P. \& ZUCCHI, R. A. (eds).
Trichogramma e o Controle Biológico Aplicado. Piracicaba: FEALQ, 1997. p.121-150.

PEREIRA, F. F.; BARROS R.; PRATISSOLI, D.; PARRA, J. R. P. Biologia e exigências térmicas de Trichogramma pretiosum Riley e $T$. exiguum Pinto \& Planter (Hymenoptera: Trichogrammatidae) criados em ovos de Plutella xylostella (L.) (Lepidoptera: Plutellidae). Neotropical Entomology. Vacaria, v.33, n.1, p. 231-236, 2004.

PRATISSOLI, D.; PARRA J. R. P. Fertility life table of Trichogramma pretiosum (Hymenopera: Trichogrammatidae) in eggs of Tuta absoluta (Lep.: Gelechiidae) at different temperatures. Journal applied Entomology. Berlin, v.124, p. 339-342, 2000.

PRATISSOLI, D.; FERNADES, O. A.; ZANUNCIO, J. C.; PASTORI, P. L. Fertility life table of Trichogramma pretiosum and Trichogramma acacioi (Hymenopera: Trichogrammatidae) on Sitotroga cerealella (Lepidopera: Gelechiidae) eggs at different constant temperatures. Annals of the Entomological Society of America. Lanham, v.97, n. 3, p. 729-731, 2004b.

PRATISSOLI, D.; ZANUNCIO J. C.; VIANNA U. R.; ANDRADE, J. S.; E. GUIMARÃES M.; ESPINDULA, M. C. Fertility life table of Trichogramma pretiosum and Trichogramma acacioi on eggs of Anagasta kuehniella at different temperatures. Pesquisa agropecuária Brasileira. Brasília, v.39, n.2, p.193-196, 2004a.

PREZOTTI, L.; PARRA, J. R. P. Controle de qualidade em criações massais de parasitóides e predadores. In: PARRA, J. R., P. S. M. BOTELHO, B. S. C. FERREIRA \& J. M. S. BENTO. Controle biológico no Brasil: Parasitóides e Predadores. São Paulo: Manole. 2002. p.295-311.

QUERINO, R. B.; ZUCCHI, R. A. New species of Trichogramma Westwood (Hymenoptera: Trichogrammatidae) associated with lepidopterous eggs in Brazil. Zootaxa. New Zealand, v.163, n. 1, p. 1-10, 2003.

SILVEIRA NETO, S.; NAKANO O.; BARBIN, D.; VILLA NOVA, N. A. Manual de ecologia de insetos. São Paulo: Ed. Ceres, 1976. 419p.

ZHANG, W. Q.; AGAMY, E.; HASSAN, S. A. Life-table characteristics of four candidates species of the genus Trichogramma to control the diamondback moth Plutella xylostella (L.). Journal of Plant Diseases and Protection. Berlin, v.108, n. 2, p.413-418, 2001. 